\title{
On the largest eigenvalue of a symmetric nonnegative tensor
}

\author{
Guanglu Zhou ${ }^{1, *, \dagger}$, Liqun $\mathrm{Qi}^{2}$ and Soon-Yi Wu ${ }^{3}$ \\ ${ }^{1}$ Department of Mathematics and Statistics, Curtin University, Perth, Australia \\ ${ }^{2}$ Department of Applied Mathematics, The Hong Kong Polytechnic University, Hong Kong \\ ${ }^{3}$ Institute of Applied Mathematics, National Cheng-Kung University, Tainan, Taiwan
}

\section{SUMMARY}

In this paper, some important spectral characterizations of symmetric nonnegative tensors are analyzed. In particular, it is shown that a symmetric nonnegative tensor has the following properties: (i) its spectral radius is zero if and only if it is a zero tensor; (ii) it is weakly irreducible (respectively, irreducible) if and only if it has a unique positive (respectively, nonnegative) eigenvalue-eigenvector; (iii) the minimax theorem is satisfied without requiring the weak irreducibility condition; and (iv) if it is weakly reducible, then it can be decomposed into some weakly irreducible tensors. In addition, the problem of finding the largest eigenvalue of a symmetric nonnegative tensor is shown to be equivalent to finding the global solution of a convex optimization problem. Subsequently, algorithmic aspects for computing the largest eigenvalue of symmetric nonnegative tensors are discussed. Copyright (C) 2013 John Wiley \& Sons, Ltd.

Received 8 November 2011; Revised 11 February 2013; Accepted 1 April 2013

KEY WORDS: eigenvalue; symmetric tensor; algorithm; convergence; convex optimization

\section{INTRODUCTION}

Let $\Re$ be the real field. In this paper, we consider an $m$-order $n$-dimensional tensor $\mathcal{A}$ consisting of $n^{m}$ entries in $\mathfrak{R}$ :

$$
\mathcal{A}=\left(a_{i_{1} i_{2} \ldots i_{m}}\right), \quad a_{i_{1} i_{2} \ldots i_{m}} \in \Re, \quad 1 \leqslant i_{1}, i_{2}, \ldots, i_{m} \leqslant n .
$$

$\mathcal{A}$ is called nonnegative (or, respectively, positive) if $a_{i_{1} i_{2} \ldots i_{m}} \geqslant 0$ (or, respectively, $a_{i_{1} i_{2} \ldots i_{m}}>0$ ). When $m=2, \mathcal{A}$ is a matrix. When $m \geqslant 3, \mathcal{A}$ is called a higher-order tensor. Tensors play an important role in physics, engineering, and mathematics. Applications of tensors include data analysis and mining, information science, signal and image processing, and computational biology, and so on. See $[1-3]$ and references therein.

To an $n$-dimensional column vector $\boldsymbol{x}=\left[\boldsymbol{x}_{1}, \boldsymbol{x}_{2}, \ldots, \boldsymbol{x}_{n}\right]^{T} \in \mathfrak{\Re}^{n}$, real or complex, we define an $n$-dimensional column vector:

$$
\mathcal{A} \boldsymbol{x}^{m-1}:=\left(\sum_{i_{2}, \ldots, i_{m}=1}^{n} a_{i i_{2} \ldots i_{m}} \boldsymbol{x}_{i_{2}} \cdots \boldsymbol{x}_{i_{m}}\right)_{1 \leqslant i \leqslant n} .
$$

Definition 1.1

Let $\mathcal{A}$ be an $m$-order $n$-dimensional tensor and $\mathcal{C}$ be the set of all complex numbers. Assume that $\mathcal{A} \boldsymbol{x}^{m-1}$ is not identical to 0 . We say that $(\lambda, \boldsymbol{x}) \in \mathcal{C} \times\left(\mathcal{C}^{n} \backslash\{0\}\right)$ is an eigenvalue-eigenvector of $\mathcal{A}$ if

$$
\mathcal{A} \boldsymbol{x}^{m-1}=\lambda \boldsymbol{x}^{[m-1]} .
$$

\footnotetext{
*Correspondence to: Guanglu Zhou, Department of Mathematics and Statistics, Curtin University, Perth, Australia.

†E-mail: G.Zhou@curtin.edu.au
} 
Here, $x^{[\alpha]}=\left[x_{1}^{\alpha}, x_{2}^{\alpha}, \ldots, x_{n}^{\alpha}\right]^{T}$.

This definition was introduced in [4-6]. Unlike matrices, eigenvalue problems for high-order tensors are nonlinear. Applications of eigenvalues of high-order tensors include medical resonance imaging [7, 8], higher-order Markov chains [9], positive definiteness of even-order multivariate forms in automatical control [10], and best-rank one approximation in data analysis [1,11,12], and so on.

Recently, eigenvalue problems for high-order tensors have gained special attention in the realm of numerical multilinear algebra. In particular, the Perron-Frobenius theorem for eigenvalues of nonnegative tensors has been established in [4, 13, 14]. Friedland, Gaubert, and Han [15] established the Perron-Frobenius theorem for homogeneous monotone maps. The Perron-Frobenius theorem for nonnegative tensors is related to measuring higher-order connectivity in linked objects [16] and hyper-graphs [17-19]. Subsequently, on the basis of the minimax theorem for nonnegative tensors in [4], Ng, Qi, and Zhou [9] proposed a power-type method for computing the largest eigenvalue and the corresponding eigenvector for nonnegative tensors. This method is an extension of a method of Collatz [20,21] for calculating the spectral radius of a nonnegative matrix. The convergence of the Ng-Qi-Zhou method [9] for primitive (respectively weakly primitive) nonnegative tensors has been established in [22] (respectively, [15]). In [23-25], the authors proposed an updated version of the Ng-Qi-Zhou method [9], and it has been proven that this algorithm is always convergent for any irreducible nonnegative tensor. Recently, it has been shown in [26] that this algorithm is convergent for any weakly irreducible nonnegative tensor. Furthermore, global linear convergence of the $\mathrm{Ng}-\mathrm{Qi}-\mathrm{Zhou}$ method and its updated version has been studied in [24,26,27].

The main purpose of this paper is to analyze some important spectral characterizations of symmetric nonnegative tensors. After some preliminaries on nonnegative tensors in Section 2, we give in Section 3 some important properties of the largest eigenvalue of a symmetric nonnegative tensor $\mathcal{A}$, including (i) the spectral radius of $\mathcal{A}$ is 0 if and only if $\mathcal{A}=0$; (ii) $\mathcal{A}$ is weakly irreducible if and only if $\mathcal{A}$ has a unique positive eigenvalue-eigenvector; (iii) $\mathcal{A}$ is irreducible if and only if $\mathcal{A}$ has a unique nonnegative eigenvalue-eigenvector; (iv) if $\mathcal{A}$ is weakly reducible, then $\mathcal{A}$ can be decomposed into some weakly irreducible tensors; and (v) the minimax theorem is satisfied without requiring the weak irreducibility condition. Some counterexamples are given to show that these properties may not be satisfied if tensor $\mathcal{A}$ is not symmetric. In addition, we show that the problem of finding the largest eigenvalue for symmetric tensors is equivalent to finding the global solution of a convex optimization problem. Subsequently, we discuss in Section 4 some algorithmic aspects for the largest eigenvalue of any symmetric nonnegative tensor.

Throughout this paper, we use $\Re^{n}\left(\mathcal{C}^{n}\right)$ to denote the $n$-dimensional real (complex) space, respectively. Let $\boldsymbol{P}_{n}=\left\{\boldsymbol{x} \in \Re^{n}: \boldsymbol{x}_{i} \geqslant 0,1 \leqslant i \leqslant n\right\}$ and $\operatorname{int}\left(\boldsymbol{P}_{n}\right)=\left\{\boldsymbol{x} \in \Re^{n}: \boldsymbol{x}_{i}>0,1 \leqslant i \leqslant n\right\}$. Matrices are denoted by italic capitals $(\boldsymbol{A}, \boldsymbol{B}, \ldots)$, and high-order tensors are written as calligraphic capitals $(\mathcal{A}, \mathcal{B}, \ldots)$.

\section{PRELIMINARIES}

In this section, we gather some definitions and results about nonnegative matrices and nonnegative tensors, which will be used in the following sections.

\subsection{Nonnegative matrices}

Let $\boldsymbol{M}$ be an $n \times n$ nonnegative matrix. The graph associated to $\boldsymbol{M}$ (see Chapter 2, [21]), $\mathcal{G}(\boldsymbol{M})$, is the directed graph with vertices $1,2, \ldots, n$ and an edge from $i$ to $j$ if and only if $\boldsymbol{M}_{i j} \neq 0$. A directed graph is said to be strongly connected if there is a directed path between any two distinct vertices. The matrix $\boldsymbol{M}$ is called irreducible if the graph $\mathcal{G}(\boldsymbol{M})$ is strongly connected. We say that $\boldsymbol{M}$ is primitive if the graph $\mathcal{G}(\boldsymbol{M})$ is strongly connected and the greatest common divisor of the lengths of its circuits is equal to one. An irreducible matrix with a nonzero main diagonal is primitive (Corollary 3.2, [28]). Let $\sigma(\boldsymbol{M})$ denote the spectrum of $\boldsymbol{M}$, the set of all eigenvalues of $\boldsymbol{M}$. The spectral radius of $\boldsymbol{M}$, denoted by $\rho(\boldsymbol{M})$, is the maximum distance of an eigenvalue from the origin, 
that is, $\rho(\boldsymbol{M})=\max \{|\lambda|: \lambda \in \sigma(\boldsymbol{M})\}$. The classical Perron-Frobenius theorem for nonnegative matrices may be stated as follows (see Chapter 2, [21]).

Theorem 2.1

If $\boldsymbol{M}$ is an irreducible nonnegative matrix, then $\boldsymbol{M}$ has an eigenvector $\boldsymbol{u} \in \operatorname{int}\left(\boldsymbol{P}_{n}\right)$, unique up to a scale multiple, whose associated eigenvalue is the spectral radius of $\boldsymbol{M}, \rho(\boldsymbol{M})$. Moreover, $\rho(\boldsymbol{M})$ is a simple root of the characteristic equation of $\boldsymbol{M}$. Furthermore, if the nonnegative matrix $\boldsymbol{M}$ is primitive, then

$$
\rho(\boldsymbol{M})>|\lambda|, \quad \forall \lambda \in \sigma(\boldsymbol{M}) \backslash\{\rho(\boldsymbol{M})\} .
$$

\subsection{Nonnegative tensors}

Let $\mathcal{A}$ be an $m$-order $n$-dimensional nonnegative tensor. The spectral radius of $\mathcal{A}$ is defined as $\rho(\mathcal{A})=\max \{|\lambda|: \lambda$ is an eigenvalue of $\mathcal{A}\}$.

Definition $2.2([4])$

An $m$-order $n$-dimensional tensor $\mathcal{A}$ is called reducible if there exists a nonempty proper index subset $I \subset\{1,2, \ldots, n\}$ such that

$$
a_{i_{1} i_{2} \ldots i_{m}}=0, \quad \forall i_{1} \in I, \quad \forall i_{2}, \ldots, i_{m} \notin I .
$$

If $\mathcal{A}$ is not reducible, then we call $\mathcal{A}$ irreducible.

Let $\mathcal{A}$ be an $m$-order $n$-dimensional nonnegative tensor. The graph associated to $\mathcal{A}, \mathcal{G}(\mathcal{A})$, is the directed graph with vertices $1,2, \ldots, n$ and an edge from $i$ to $j$ if and only if $a_{i} i_{2} \ldots i_{m} \neq 0$ for some $i_{l}=j, l=2,3, \ldots, m$.

Definition 2.3 ([15])

An $m$-order $n$-dimensional tensor $\mathcal{A}$ is called weakly irreducible if $\mathcal{G}(\mathcal{A})$ is strongly connected. If $\mathcal{G}(\mathcal{A})$ is strongly connected and the greatest common divisor of the lengths of its circuits is equal to 1 , then $\mathcal{A}$ is called weakly primitive.

We have the following proposition.

Proposition 2.1 ([15])

If the nonnegative tensor $\mathcal{A}$ is irreducible, then $\mathcal{A}$ is weakly irreducible. For $m=2, \mathcal{A}$ is irreducible if and only if $\mathcal{A}$ is weakly irreducible.

Let $\mathcal{I}$ be the $m$-order $n$-dimensional unit tensor whose entries are

$$
I_{i_{1} i_{2} \ldots i_{m}}= \begin{cases}1 & \text { if } i_{1}=i_{2}=\ldots=i_{m} \\ 0 & \text { otherwise }\end{cases}
$$

Proposition 2.2 ([26])

If the nonnegative tensor $\mathcal{A}$ is weakly irreducible, then $\mathcal{A}+\mathcal{I}$ is weakly primitive.

Let $\mathcal{A}$ be a nonnegative tensor. For any vector $\boldsymbol{x} \in \boldsymbol{P}_{n}$, we define the following sequence $\left\{\mathcal{A}^{(k)} \boldsymbol{x}\right\}$ :

$$
\begin{aligned}
\mathcal{A}^{(1)} \boldsymbol{x} & =\mathcal{A}(\boldsymbol{x})^{m-1}, z^{(1)}=\left(\mathcal{A}^{(1)} \boldsymbol{x}\right)^{\left[\frac{1}{m-1}\right]} \\
\mathcal{A}^{(2)} \boldsymbol{x} & =\mathcal{A}\left(z^{(1)}\right)^{m-1}, z^{(2)}=\left(\mathcal{A}^{(2)} \boldsymbol{x}\right)^{\left[\frac{1}{m-1}\right]} \\
& \vdots \\
\mathcal{A}^{(k)} \boldsymbol{x} & =\mathcal{A}\left(z^{(k-1)}\right)^{m-1}, z^{(k)}=\left(\mathcal{A}^{(k)} \boldsymbol{x}\right)^{\left[\frac{1}{m-1}\right]}, k \geqslant 2 .
\end{aligned}
$$


Definition 2.4 ([22])

A nonnegative tensor $\mathcal{A}$ is primitive if there exists a positive integer $k$ such that $\mathcal{A}^{(k)} \boldsymbol{x} \in \operatorname{int}\left(\boldsymbol{P}_{n}\right)$ for any nonzero $\boldsymbol{x} \in \boldsymbol{P}_{n}$.

Clearly, positive tensors and essentially positive tensors [22,29] are primitive. A primitive nonnegative tensor $\mathcal{A}$ is irreducible, but the converse is false [22]. We have the following result.

Theorem $2.2([22,23])$

Suppose $\mathcal{A}$ is an irreducible nonnegative tensor. Let $\mathcal{B}=\mathcal{A}+\mathcal{I}$. Then,

(i) $\mathcal{B}$ is primitive.

(ii) If $\lambda$ is the largest eigenvalue of $\mathcal{B}$ and $\boldsymbol{u}$ is a positive eigenvector of $\mathcal{B}$ associated with $\lambda$, then $\lambda-1$ is the largest eigenvalue of $\mathcal{A}$, and $\boldsymbol{u}$ is a positive eigenvector of $\mathcal{A}$ associated with $\lambda-1$.

In $[4,15]$, the Perron-Frobenius theorem and the well-known Collatz [20] minimax theorem for nonnegative matrices have been extended to nonnegative tensors, and further results for PerronFrobenius theorem for nonnegative tensors have been given in [30,31]. In the following, we state these results for reference.

Theorem 2.3

Let $\mathcal{A}$ be a nonnegative tensor of order $m$ and dimension $n$ and $\rho(\mathcal{A})$ be the spectral radius of $\mathcal{A}$.

(i) [30] $\rho(\mathcal{A})$ is an eigenvalue of $\mathcal{A}$ with a nonnegative eigenvector.

(ii) [15] If $\mathcal{A}$ is weakly irreducible, then $\mathcal{A}$ has a positive eigenvector $\boldsymbol{u} \in \operatorname{int}\left(\boldsymbol{P}_{n}\right)$, unique up to a scale multiple, whose associated eigenvalue is $\rho(\mathcal{A})$.

(iii) [4] If $\mathcal{A}$ is irreducible, then $\mathcal{A}$ has a positive eigenvector $\boldsymbol{u} \in \operatorname{int}\left(\boldsymbol{P}_{n}\right)$ whose associated eigenvalue is $\rho(\mathcal{A})$. Moreover, if $\lambda$ is an eigenvalue with nonnegative eigenvector, then $\lambda=\rho(\mathcal{A})$, and the nonnegative eigenvector is unique up to a multiplicative constant.

\subsection{The Ng-Qi-Zhou algorithm}

In this subsection, we state a power-type algorithm for calculating the largest eigenvalue of a nonnegative tensor $\mathcal{A}$ by applying the algorithm proposed by $\mathrm{Ng}$, Qi, and Zhou in [9] to tensor $\mathcal{B}=\mathcal{A}+\mathcal{I}$ and establish the Q-linear convergence of this power algorithm under a weak irreducibility condition. This algorithm has been studied in [23].

For any nonnegative column vector $\boldsymbol{x} \in \mathfrak{\Re}^{n}$, we define $\phi: \boldsymbol{P}_{n} \rightarrow P_{1}$ by

$$
\phi(x)=\sum_{i=1}^{n} x_{i} .
$$

Algorithm 2.1

Step 0. Choose $\boldsymbol{x}^{(1)} \in \operatorname{int}\left(\boldsymbol{P}_{n}\right)$. Let $\mathcal{B}=\mathcal{A}+\mathcal{I}$, and set $k:=1$.

Step 1. Compute

$$
\begin{aligned}
y^{(k)} & =\mathcal{B}\left(\boldsymbol{x}^{(k)}\right)^{m-1}, \\
\underline{\lambda}_{k} & =\min _{\boldsymbol{x}_{i}^{(k)}>0} \frac{\left(y^{(k)}\right)_{i}}{\left(\boldsymbol{x}_{i}^{(k)}\right)^{m-1}}, \\
\bar{\lambda}_{k} & =\max _{\boldsymbol{x}_{i}^{(k)}>0} \frac{\left(y^{(k)}\right)_{i}}{\left(\boldsymbol{x}_{i}^{(k)}\right)^{m-1}} .
\end{aligned}
$$


Step 2. If $\bar{\lambda}_{k}=\underline{\lambda}_{k}$, then let $\lambda=\bar{\lambda}_{k}$, and stop. Otherwise, compute

$$
\boldsymbol{x}^{(k+1)}=\frac{\left(y^{(k)}\right)^{\left[\frac{1}{m-1}\right]}}{\phi\left(\left(y^{(k)}\right)^{\left[\frac{1}{m-1}\right]}\right)},
$$

replace $k$ by $k+1$, and go to Step 1 .

Let

$$
F(\boldsymbol{x})=\mathcal{B} \boldsymbol{x}^{m-1}, \quad G(\boldsymbol{x})=F(\boldsymbol{x})^{\left[\frac{1}{m-1}\right]} \text { and } H(\boldsymbol{x})=\frac{G(\boldsymbol{x})}{\phi(G(\boldsymbol{x}))} .
$$

Clearly, the sequence $\left\{\boldsymbol{x}^{(k)}\right\}$ generated by Algorithm 2.1 satisfies

$$
\boldsymbol{x}^{(k+1)}=H\left(\boldsymbol{x}^{(k)}\right), k=1,2, \ldots,
$$

and $\phi\left(\boldsymbol{x}^{(k)}\right)=1$ for all $k=1,2, \cdots$.

Theorem $2.4([22,23])$

Suppose the nonnegative tensor $\mathcal{A}$ is irreducible and $(\rho(\mathcal{A})$; $\mathbf{u})$ is a positive eigenvalue-eigenvector of $\mathcal{A}$ satisfying $\phi(\mathbf{u})=1$. Then, starting from any $\boldsymbol{x}^{(1)} \in \boldsymbol{P} \backslash\{0\}$, Algorithm 2.1 produces a value of $\lambda$ and a corresponding eigenvector $\boldsymbol{u}$ in a finite number of steps or generates three convergent sequences $\left\{\underline{\lambda}_{k}\right\},\left\{\bar{\lambda}_{k}\right\}$, and $\left\{\boldsymbol{x}^{(k)}\right\}$ such that $\lim _{k \rightarrow \infty} \underline{\lambda}_{k}=\lim _{k \rightarrow \infty} \bar{\lambda}_{k}=\lambda$ and $\lim _{k \rightarrow \infty} \boldsymbol{x}^{(k)}=\mathbf{u}$. Furthermore, $\rho(\mathcal{A})=\lambda-1$.

Theorem $2.5([15,26])$

Suppose the nonnegative tensor $\mathcal{A}$ is weakly irreducible and $(\rho(\mathcal{A})$, u) is a positive eigenvalueeigenvector of $\mathcal{A}$ satisfying $\phi(\mathbf{u})=1$. Then, starting from any $\boldsymbol{x}^{(1)} \in \operatorname{int}(\boldsymbol{P})$, Algorithm 2.1 generates a sequence $\left\{\boldsymbol{x}^{(k)}\right\}$, which converges to the vector $\mathbf{u}$.

Proof

By Proposition 2.2, $\mathcal{B}$ is weakly primitive. Hence, from Corollary 5.1 [15] and the result (ii) of Theorem 2.2, this theorem holds.

Next, we give the Q-linear convergence of Algorithm 2.1.

\section{Theorem 2.6}

Let $\mathcal{A}, \mathcal{B}$, and $\left\{\boldsymbol{x}^{(k)}\right\}$ be as in Theorem 2.5. If $\mathcal{A}$ is weakly irreducible, then the sequence $\left\{\boldsymbol{x}^{(k)}\right\}$ is convergent, and the convergence rate of $\left\{\boldsymbol{x}^{(k)}\right\}$ is Q-linear, that is, there exists a vector norm $\|\cdot\|$ such that

$$
\limsup _{k \rightarrow \infty} \frac{\left\|\boldsymbol{x}^{(k+1)}-\boldsymbol{u}\right\|}{\left\|\boldsymbol{x}^{(k)}-\boldsymbol{u}\right\|}<1
$$

Theorem 2.6 can be proved in a similar argument as in Corollary 5.2 [15], so we omit it. In Theorem 2.6, we establish the Q-linear convergence of Algorithm 2.1 under the weak irreducibility condition. A power algorithm for polynomial eigenvalue problems has been recently introduced in [15], and the R-linear convergence of the power algorithm has been established under the weak primitivity condition. In addition, it is shown recently in [26] that Algorithm 2.1 is globally R-linearly convergent for weakly irreducible nonnegative tensors. 


\section{SYMMETRIC NONNEGATIVE TENSORS}

In this section, we give some important properties of symmetric nonnegative tensors; see Theorems 3.1-3.5. In addition, we show that the largest eigenvalue of a symmetric nonnegative tensor connects with the global solution of a convex polynomial optimization problem.

Tensor $\mathcal{A}$ is called symmetric if its entries $a_{i_{1}} i_{2} \ldots i_{m}$ are invariant under any permutation of their indices $\left\{i_{1}, i_{2}, \ldots, i_{m}\right\}$ [6]. Let $f(\boldsymbol{x})$, an $m$ th degree homogeneous polynomial form of $n$ variables, be defined by

$$
f(\boldsymbol{x}):=\mathcal{A} \boldsymbol{x}^{m}=\sum_{i_{1}, i_{2}, \ldots, i_{m}=1}^{n} a_{i_{1} i_{2} \ldots i_{m}} \boldsymbol{x}_{i_{1}} \boldsymbol{x}_{i_{2}} \cdots \boldsymbol{x}_{i_{m}} .
$$

By simple computation,

$$
\nabla f(\boldsymbol{x}):=m \mathcal{A} \boldsymbol{x}^{m-1}=m\left(\sum_{i_{2}, \ldots, i_{m}=1}^{n} a_{i i_{2} \ldots i_{m}} \boldsymbol{x}_{i_{2}} \cdots \boldsymbol{x}_{i_{m}}\right)_{1 \leqslant i \leqslant n} .
$$

We consider the following constrained optimization problem:

$$
\begin{aligned}
\max & f(\boldsymbol{x}) \\
\text { s.t } & \sum_{i=1}^{n} \boldsymbol{x}_{i}^{m}=1 \\
& \boldsymbol{x} \geqslant 0 .
\end{aligned}
$$

A generalized form of Problem (3.10) has been extensively studied in [32], and it is shown in [32] that this generalized optimization problem can be used for solving some hardly tractable combinational optimization problems such as the labeling problem.

\section{Lemma 3.1}

Suppose the nonnegative tensor $\mathcal{A}$ is symmetric. If $\boldsymbol{x}$ is a local solution of Problem (3.10), then $(f(\boldsymbol{x}), \boldsymbol{x})$ is a nonnegative eigenvalue-eigenvector of $\mathcal{A}$.

Proof

If $\boldsymbol{x}$ is a local solution of Problem (3.10), then, by Theorem 12.1 of [33], there exist $\boldsymbol{c} \in \Re$ and $z \in \mathfrak{\Re}^{n}$ such that

$$
\begin{aligned}
\mathcal{A} \boldsymbol{x}^{m-1} & =\boldsymbol{c} \boldsymbol{x}^{[m-1]}-z, \\
\boldsymbol{x}_{i} z_{i} & =0, \boldsymbol{x}_{i} \geqslant 0, z_{i} \geqslant 0, i=1,2, \ldots, n, \\
\sum_{i=1}^{n} \boldsymbol{x}_{i}^{m} & =1 .
\end{aligned}
$$

From (3.11)-(3.13), we have

$$
f(x)=\mathcal{A} x^{m}=x^{T}\left(\mathcal{A} x^{m-1}\right)=c x^{T}\left(x^{[m-1]}\right)-x^{T} z=c \sum_{i=1}^{n} x_{i}^{m}=c .
$$

For $i=1,2, \ldots, n$, by (3.12), $z_{i}=0$ if $\boldsymbol{x}_{i}>0$. If $\boldsymbol{x}_{i}=0$, by (3.11), $\left(\mathcal{A} \boldsymbol{x}^{m-1}\right)_{i}=-\boldsymbol{z}_{i}$. Because $\left(\mathcal{A} \boldsymbol{x}^{m-1}\right)_{i} \geqslant 0$ and $z_{i} \geqslant 0$, we obtain $z_{i}=0$. Hence, $z_{i}=0$ for all $i=1,2, \ldots, n$. Therefore, from $(3.11), \mathcal{A} \boldsymbol{x}^{m-1}=\boldsymbol{c} \boldsymbol{x}^{[m-1]}$, which implies that $(f(\boldsymbol{x}), \boldsymbol{x})=(\boldsymbol{c}, \boldsymbol{x})$ is a nonnegative eigenvalue-eigenvector of $\mathcal{A}$.

Definition 3.5

$(\lambda, \boldsymbol{x})$ is called a KKT pair of Problem (3.10) if it satisfies

$$
\mathcal{A} x^{m-1}=\lambda \boldsymbol{x}^{[m-1]}, \sum_{i=1}^{n} \boldsymbol{x}_{i}^{m}=1, \lambda \geqslant 0, \boldsymbol{x} \geqslant 0 .
$$


Clearly, $(\lambda, \boldsymbol{x})$ is a KKT pair of Problem (3.10) if and only if $(\lambda, \boldsymbol{x})$ is a nonnegative eigenvalue-eigenvector of $\mathcal{A}$.

Theorem 3.1

Let $\mathcal{A}$ be a symmetric nonnegative tensor. Then, $\rho(\mathcal{A})=0$ if and only if $\mathcal{A}=0$.

Proof

Clearly, if $\mathcal{A}=0$, then $\rho(\mathcal{A})=0$. Suppose $\mathcal{A} \neq 0$. Then, there exists $\boldsymbol{x} \in \mathfrak{\Re}^{n}$ satisfying $\sum_{i=1}^{n} \boldsymbol{x}_{i}^{m}=1$ such that $f(\boldsymbol{x})=\mathcal{A} \boldsymbol{x}^{m}>0$. This means that there exists $\boldsymbol{y} \in \mathfrak{R}^{n}$ satisfying $f(\boldsymbol{y})>0$ such that $\boldsymbol{y}$ is a local solution of Problem (3.10). By Lemma 3.1, $(f(\boldsymbol{y}), \boldsymbol{y})$ is a nonnegative eigenvalue-eigenvector of $\mathcal{A}$. Hence, $\rho(\mathcal{A}) \geqslant f(\boldsymbol{y})>0$. Therefore, if $\rho(\mathcal{A})=0$, then $\mathcal{A}=0$. This completes the proof.

\section{Remark 1}

Theorem 3.1 is not true if $\mathcal{A}$ is not symmetric. Consider a three-order two-dimensional tensor $\mathcal{F}$ given by $f_{121}=1$ and 0 elsewhere. By simple computation, we obtain $\rho(\mathcal{F})=0$, but $\mathcal{F} \neq 0$. In [26], it is shown that $\rho(\mathcal{T})>0$ if the nonnegative tensor $\mathcal{T}$ is strictly nonnegative. In Theorem 3.1, it is shown that if the nonnegative tensor $\mathcal{T}$ is symmetric and $\mathcal{T} \neq 0$, then $\rho(\mathcal{T})>0$.

Definition 3.6 ([32])

A homogeneous polynomial function $f(\boldsymbol{x})$ defined in (3.8) is called reducible if there exists a nonempty proper index subset $I \subset\{1,2, \ldots, n\}$ such that

$$
f(\boldsymbol{x})=f_{1}\left(\boldsymbol{x}_{I}\right)+f_{2}\left(\boldsymbol{x}_{J}\right),
$$

where $J=\{1,2, \ldots, n\} \backslash I, f_{1}\left(x_{I}\right)$, and $f_{2}\left(x_{J}\right)$ are homogeneous polynomial functions, respectively. If $f(\boldsymbol{x})$ is not reducible, then we call $f(\boldsymbol{x})$ irreducible.

We have the following result.

\section{Lemma 3.2}

Let $f(\boldsymbol{x})$ and $\mathcal{A}$ be as in (3.8). Then, $\mathcal{A}$ is weakly irreducible if and only if $f(\boldsymbol{x})$ is irreducible.

\section{Proof}

Suppose $\mathcal{A}$ is weakly irreducible, and assume to the contrary that $f(\boldsymbol{x})$ is reducible. Then, there exists a partition $\{I, J\}$ of $\{1,2, \ldots, n\}$ such that $f(\boldsymbol{x})=f_{1}\left(\boldsymbol{x}_{I}\right)+f_{2}\left(\boldsymbol{x}_{J}\right)$. This implies that $a_{i_{1} i_{2} \ldots i_{m}}=0$ if $i_{1} \in I$ and some $i_{j} \in J, j=2,3, \ldots, m$, and $a_{i_{1} i_{2} \ldots i_{m}}=0$ if $i_{1} \in J$, and some $i_{j} \in I, j=2,3, \ldots, m$. Hence, the graph associated to $\mathcal{A}, \mathcal{G}(\mathcal{A})$, is not strongly connected because there is not an edge for any $i \in I$ and $j \in J$, which contradicts the weak irreducibility of $\mathcal{A}$.

Now we suppose $f(\boldsymbol{x})$ is irreducible and assume to the contrary that $\mathcal{A}$ is weakly reducible. By Definition 2.3, the graph associated to $\mathcal{A}, \mathcal{G}(\mathcal{A})$, is not strongly connected. Then, there exists a partition $\{I, J\}$ of $\{1,2, \ldots, n\}$ such that there is not an edge for any $i \in I$ and $j \in J$. This implies that $a_{i_{1} i_{2} \ldots i_{m}}=0$ if there exist some $i_{k} \in I$ and $i_{j} \in J, k \neq j, k, j=1,2, \ldots, m$. Hence, by (3.8), $f(\boldsymbol{x})$ can be written as the sum of two functions $f_{1}\left(x_{I}\right)$ and $f_{2}\left(x_{J}\right)$, which contradicts the irreducibility of $f(\boldsymbol{x})$.

Let $\mathcal{A}$ be a nonnegative tensor of order $m$ and dimension $n$, and $I \subset\{1,2, \ldots, n\}$. We define $\mathcal{A}_{I}=\left(d_{i_{1} i_{2} \ldots i_{m}}\right), i_{j} \in I, j=1,2, \ldots, m$, a tensor induced from $\mathcal{A}$, by

$$
d_{i_{1} i_{2} \ldots i_{m}}=a_{i_{1} i_{2} \ldots i_{m}}, i_{j} \in I, j=1,2, \ldots, m .
$$

Let $|I|$ be the number of elements of $I$. Then, $\mathcal{A}_{I}$ is a tensor of order $m$ and dimension $|I|$.

By using Lemma 3.2, it is easy to obtain that if $\mathcal{A}$ is weakly reducible, then there exists a partition $\left\{I_{1}, I_{2}, \ldots, I_{k}\right\}$ of $\{1,2, \ldots, n\}$ such that the function $f(\boldsymbol{x})$ defined in (3.8) can be written as

$$
f(\boldsymbol{x})=\sum_{j=1}^{k} f_{j}\left(\boldsymbol{x}_{I_{j}}\right),
$$


where for each $j=1,2, \ldots, k, f_{j}\left(\boldsymbol{x}_{I_{j}}\right)$ is irreducible or it is a zero function. Moreover, for each $j=1,2, \ldots, k$, the corresponding induced tensor $\mathcal{A}_{I_{j}}$ is either weakly irreducible or a zero tensor. From this observation, we have the following theorem.

Theorem 3.2

Let $\mathcal{A}$ be a symmetric nonnegative tensor of order $m$ and dimension $n$. Suppose $\mathcal{A}$ is weakly reducible. Then, we have the following results.

(1) There exists a partition $\left\{I_{1}, I_{2}, \ldots, I_{k}\right\}$ of $\{1,2, \ldots, n\}$ such that each induced tensor $\mathcal{A}_{I_{i}}, i=$ $1,2, \ldots, k$ is either weakly irreducible or a zero tensor.

(2) There exists an $n \times n$ permutation matrix $\boldsymbol{P}$ such that

$$
\boldsymbol{P}\left(\mathcal{A} \boldsymbol{x}^{m-1}\right)=\left[\begin{array}{c}
\mathcal{A}_{I_{1}}\left(\boldsymbol{x}_{I_{1}}\right)^{m-1} \\
\mathcal{A}_{I_{2}}\left(\boldsymbol{x}_{I_{2}}\right)^{m-1} \\
\vdots \\
\mathcal{A}_{I_{k}}\left(\boldsymbol{x}_{I_{k}}\right)^{m-1}
\end{array}\right] .
$$

(3) The eigenvalues of $\mathcal{A}_{I_{i}}, i=1,2, \ldots, k$ are the eigenvalues of $\mathcal{A}$. If $(\lambda, \boldsymbol{x}) \in \mathcal{C} \times\left(\mathcal{C}^{\boldsymbol{n}} \backslash\{\mathbf{0}\}\right)$ is an eigenvalue-eigenvector of $\mathcal{A}$, then $\lambda$ is an eigenvalue of some $\mathcal{A}_{I_{i}}, 1 \leqslant i \leqslant k$.

(4) $\rho(\mathcal{A})=\max _{1 \leqslant i \leqslant k} \rho\left(\mathcal{A}_{I_{i}}\right)$.

Proof

It follows from (3.15) that (1) holds, and then (2) is satisfied. By (3.16), we can easily obtain that (3) holds. Hence, (4) is satisfied.

By Theorem 3.2, we have the following minimax theorem for symmetric nonnegative tensors, without the weak irreducibility condition.

Theorem 3.3

Assume that $\mathcal{A}$ is a symmetric nonnegative tensor of order $m$ and dimension $n$. Then,

$$
\operatorname{Min}_{\boldsymbol{x} \in \operatorname{int}(\boldsymbol{P})} \operatorname{Max}_{1 \leqslant i \leqslant n} \frac{\left(\mathcal{A} \boldsymbol{x}^{m-1}\right)_{i}}{\boldsymbol{x}_{i}^{m-1}}=\rho(\mathcal{A})=\operatorname{Max}_{\boldsymbol{x} \in \boldsymbol{P} \backslash\{0\}} \operatorname{Min}_{\boldsymbol{x}_{i} \neq 0,1 \leqslant i \leqslant n} \frac{\left(\mathcal{A} \boldsymbol{x}^{m-1}\right)_{i}}{\boldsymbol{x}_{i}^{m-1}} .
$$

Proof

It has been proven in $[4,15]$ that $(3.17)$ holds if $\mathcal{A}$ is weakly irreducible or it is irreducible. Now we will show that (3.17) also holds if $\mathcal{A}$ is weakly reducible. By using Theorem 3.2, if $\mathcal{A}$ is weakly reducible, then there exists a partition $\left\{I_{1}, I_{2}, \ldots, I_{k}\right\}$ of $\{1,2, \ldots, n\}$ such that each induced tensor $\mathcal{A}_{I_{i}}, i=1,2, \ldots, k$ is either weakly irreducible or a zero tensor. Moreover, without loss of generality, we suppose

$$
\mathcal{A} x^{m-1}=\left[\begin{array}{c}
\mathcal{A}_{I_{1}}\left(x_{I_{1}}\right)^{m-1} \\
\mathcal{A}_{I_{2}}\left(x_{I_{2}}\right)^{m-1} \\
\vdots \\
\mathcal{A}_{I_{k}}\left(x_{I_{k}}\right)^{m-1}
\end{array}\right] .
$$

For each $i=1,2, \ldots, k$, by Corollary 4.2 [15], we obtain

$$
\operatorname{Min}_{\boldsymbol{x}_{I_{i}>0}} \operatorname{Max}_{j \in I_{i}} \frac{\left(\mathcal{A}_{I_{i}}\left(\boldsymbol{x}_{I_{i}}\right)^{m-1}\right)_{j}}{\boldsymbol{x}_{j}^{m-1}}=\rho\left(\mathcal{A}_{I_{i}}\right)=\operatorname{Max}_{\boldsymbol{x}_{I_{i}} \geqslant 0, \boldsymbol{x}_{I_{i}} \neq 0} \operatorname{Min}_{\boldsymbol{x}_{j} \neq 0, j \in I_{i}} \frac{\left(\mathcal{A}_{I_{i}}\left(\boldsymbol{x}_{I_{i}}\right)^{m-1}\right)_{j}}{\boldsymbol{x}_{j}^{m-1}} .
$$

Note that (3.19) holds for a zero tensor. For each $\boldsymbol{x} \in \operatorname{int}(\boldsymbol{P})$, by the left equality of (3.19), we have

$$
\operatorname{Max}_{j \in I_{i}} \frac{\left(\mathcal{A}_{I_{i}}\left(\boldsymbol{x}_{I_{i}}\right)^{m-1}\right)_{j}}{\boldsymbol{x}_{j}^{m-1}} \geqslant \rho\left(\mathcal{A}_{I_{i}}\right), i=1,2, \ldots, k .
$$


So, by (3.18) and the statement (4) of Theorem 3.2,

$$
\operatorname{Max}_{1 \leqslant i \leqslant n} \frac{\left(\mathcal{A} \boldsymbol{x}^{m-1}\right)_{i}}{\boldsymbol{x}_{i}^{m-1}} \geqslant \max _{1 \leqslant i \leqslant k} \rho\left(\mathcal{A}_{I_{i}}\right)=\rho(\mathcal{A}) .
$$

Let $\boldsymbol{u}_{I_{i}}(>0)$ be an eigenvector of $\mathcal{A}_{I_{i}}$ with the associated eigenvalue $\rho\left(\mathcal{A}_{I_{i}}\right), k=1,2, \ldots, k$, and define $\boldsymbol{u}=\left(\boldsymbol{u}_{I_{i}}, 1 \leqslant i \leqslant k\right)$. Clearly, we obtain

$$
\operatorname{Max}_{1 \leqslant i \leqslant n} \frac{\left(\mathcal{A} \boldsymbol{u}^{m-1}\right)_{i}}{\boldsymbol{u}_{i}^{m-1}}=\rho(\mathcal{A})
$$

It follows from (3.20) and (3.21) that the left equality of (3.17) is satisfied. We now prove that the right equality of (3.17) holds. For each $\boldsymbol{x} \in \boldsymbol{P} \backslash\{0\}$, by the right equality of (3.19), we have

$$
\operatorname{Min}_{\boldsymbol{x}_{j} \neq 0, j \in I_{i}} \frac{\left(\mathcal{A}_{I_{i}}\left(\boldsymbol{x}_{I_{i}}\right)^{m-1}\right)_{j}}{\boldsymbol{x}_{j}^{m-1}} \leqslant \rho\left(\mathcal{A}_{I_{i}}\right) \text {, for some } 1 \leqslant i \leqslant k .
$$

So, by (3.18) and the statement (4) of Theorem 3.2,

$$
\operatorname{Min}_{\boldsymbol{x}_{i} \neq 0,1 \leqslant i \leqslant n} \frac{\left(\mathcal{A} \boldsymbol{x}^{m-1}\right)_{i}}{\boldsymbol{x}_{i}^{m-1}} \leqslant \rho\left(\mathcal{A}_{I_{i}}\right) \leqslant \rho(\mathcal{A})
$$

Let $\boldsymbol{v}_{I_{j}}(>0)$ be an eigenvector of $\mathcal{A}_{I_{j}}$ with the associated eigenvalue $\rho\left(\mathcal{A}_{I_{j}}\right)=\rho(\mathcal{A})$, and define $\boldsymbol{v} \in \mathfrak{\Re}^{n}$ by $\boldsymbol{v}_{i}=\left(\boldsymbol{v}_{I_{j}}\right)_{i}$ if $i \in I_{j}$ and zero otherwise. Clearly, we obtain

$$
\operatorname{Min}_{\boldsymbol{v}_{i} \neq 0,1 \leqslant i \leqslant n} \frac{\left(\mathcal{A} \boldsymbol{v}^{m-1}\right)_{i}}{\boldsymbol{v}_{i}^{m-1}}=\rho(\mathcal{A}) .
$$

It follows from (3.22) and (3.23) that the right equality of (3.17) is satisfied. So we completes the proof.

\section{Remark 2}

Theorem 3.3 does not hold if $\mathcal{A}$ is not symmetric. Consider a three-order two-dimensional tensor $\mathcal{S}$ given by $s_{111}=2, s_{122}=s_{222}=1$ and zero elsewhere. By simple computation, we obtain $\rho(\mathcal{S})=2$ with associated eigenvector $\boldsymbol{x}=(1,0)^{T}$. However, the left equality of (3.17) is not satisfied for tensor $\mathcal{S}$. Recently, it has been shown in Theorems 5.2 and 5.3 of [30] that the right equality of (3.17) is satisfied for any nonnegative tensor and the left equality of (3.17) holds under the condition that the nonnegative tensor $\mathcal{A}$ has a positive eigenvector corresponding to some eigenvalue. Theorem 3.3 shows that (3.17) is satisfied for any nonnegative symmetric tensor.

Lemma 3.3

Let $\mathcal{A}$ and $\mathcal{A}_{I_{i}}, i=1,2, \ldots, k$ be as in Theorem 3.2. Then, the following two statements are equivalent.

(a) $\mathcal{A}_{I_{i}}, i=1,2, \ldots, k$ are weakly irreducible and $\rho\left(\mathcal{A}_{I_{1}}\right)=\rho\left(\mathcal{A}_{I_{2}}\right)=\ldots=\rho\left(\mathcal{A}_{I_{k}}\right)$.

(b) $\mathcal{A}$ has a positive eigenvector $\boldsymbol{u} \in \operatorname{int}\left(\boldsymbol{P}_{n}\right)$ whose associated eigenvalue is $\rho(\mathcal{A})$.

\section{Proof}

By Theorem 3.2, without loss of generality, we suppose that (3.18) holds. Assume that (a) holds. Then, for each $i=1,2, \ldots, k, \mathcal{A}_{I_{i}}$ has a positive eigenvalue-eigenvector $\left(\rho\left(\mathcal{A}_{I_{i}}\right), \boldsymbol{u}_{I_{i}}\right)$. From Theorem 3.2, we obtain $\rho(\mathcal{A})=\rho\left(\mathcal{A}_{I_{1}}\right)=\rho\left(\mathcal{A}_{I_{2}}\right)=\ldots=\rho\left(\mathcal{A}_{I_{k}}\right)$. Let $\boldsymbol{u}=\left(\boldsymbol{u}_{I_{i}}, i=\right.$ $1,2, \ldots, k) \in \mathfrak{\Re}^{n}$ be formed by $\boldsymbol{u}_{I_{i}}, i=1,2, \ldots, k$. Then, by $(3.18),(\rho(\mathcal{A}), \boldsymbol{u})$ is a positive eigenvalue-eigenvector of $\mathcal{A}$.

Now, we suppose that (b) holds. Then, by $(3.18),\left(\rho(\mathcal{A}), \boldsymbol{u}_{I_{i}}\right)$ is a positive eigenvalue-eigenvector of $\mathcal{A}_{I_{i}}, i=1,2, \ldots, k$. Hence, $\mathcal{A}_{I_{i}}, i=1,2, \ldots, k$ are not zero tensors, and (a) is satisfied. 


\section{Theorem 3.4}

Let $\mathcal{A}$ be a symmetric nonnegative tensor of order $m$ and dimension $n$. Then, the following two statements are equivalent.

(i) $\mathcal{A}$ is weakly irreducible.

(ii) $\mathcal{A}$ has a positive eigenvector $\boldsymbol{u} \in \operatorname{int}\left(\boldsymbol{P}_{n}\right)$, unique up to a scale multiple, whose associated eigenvalue is $\rho(\mathcal{A})$.

Proof

If $\mathcal{A}$ is weakly irreducible, then, by Theorem 2.3, (ii) holds. Now we suppose that (ii) holds and assume to the contrary that $\mathcal{A}$ is weakly reducible. By Theorem 3.2 and Lemma 3.3, there exists a partition $\left\{I_{1}, I_{2}, \ldots, I_{k}\right\}$ of $\{1,2, \ldots, n\}$ such that each induced tensor $\mathcal{A}_{I_{i}}, i=1,2, \ldots, k$ is weakly irreducible, and $\rho(\mathcal{A})=\rho\left(\mathcal{A}_{I_{i}}\right), 1 \leqslant i \leqslant k$. Moreover, without loss of generality, we suppose that (3.18) holds. For each $i=1,2, \ldots, k, \mathcal{A}_{I_{i}}$ has a positive eigenvalue-eigenvector $\left(\rho\left(\mathcal{A}_{I_{i}}\right), \boldsymbol{u}_{I_{i}}\right)$. Let $\boldsymbol{u}=\left(\boldsymbol{u}_{I_{i}}, i=1,2, \ldots, k\right) \in \mathfrak{\Re}^{n}$ be formed by $\boldsymbol{u}_{I_{i}}, i=1,2, \ldots, k$, and $\boldsymbol{v}=\left(i \times \boldsymbol{u}_{I_{i}}, i=1,2, \ldots, k\right) \in \mathfrak{R}^{n}$. Then, by (3.18), $(\rho(\mathcal{A}), \boldsymbol{u})$ and $(\rho(\mathcal{A}), \boldsymbol{v})$ are positive eigenvalue-eigenvectors of $\mathcal{A}$, and clearly there does not exist a constant $\boldsymbol{c} \neq 0$ such that $\boldsymbol{u}=\boldsymbol{c} \boldsymbol{v}$, which contradicts (ii). So we complete the proof.

\section{Remark 3}

Theorem 3.4 does not hold if $\mathcal{A}$ is not symmetric. Consider a three-order two-dimensional tensor $\mathcal{D}$ given by $d_{122}=d_{222}=1$ and zero elsewhere. By simple computation, we obtain $\mathcal{D}$ with a positive eigenvector $\boldsymbol{x}=(1,1)^{T}$, unique up to a scale multiple, whose associated eigenvalue is 1 . However, $\mathcal{D}$ is weakly reducible.

Theorem 3.5

Let $\mathcal{A}$ be a symmetric nonnegative tensor of order $m$ and dimension $n$. Then, the following two statements are equivalent.

(a) $\mathcal{A}$ is irreducible.

(b) Problem (3.10) has a unique KKT pair $(\lambda, \boldsymbol{x})$, and $\lambda$ and $\boldsymbol{x}$ are positive.

Proof

If $\mathcal{A}$ is irreducible, then, by the statement (iii) of Theorem 2.3 and Definition 3.5, (b) holds.

Now we suppose that (b) holds and assume to the contrary that $\mathcal{A}$ is reducible, that is, there exists a nonempty proper index subset $I \subset\{1,2, \ldots, n\}$ such that

$$
a_{i_{1} i_{2} \ldots i_{m}}=0, \quad \forall i_{1} \in I, \quad \forall i_{2}, \ldots, i_{m} \in J, J=\{1,2, \ldots, n\} \backslash I .
$$

Let $\mathcal{A}_{J}$ be the induced tensor from $\mathcal{A}$ and $\boldsymbol{x}=\left(0_{I}, \boldsymbol{x}_{J}\right) \in \mathfrak{R}^{n}$. Then, it follows from (3.24) that there exists a permutation matrix $\boldsymbol{P}$ such that

$$
\boldsymbol{P}\left(\mathcal{A} \boldsymbol{x}^{m-1}\right)=\left[\begin{array}{c}
0_{I} \\
\mathcal{A}_{J}\left(\boldsymbol{x}_{J}\right)^{m-1}
\end{array}\right] .
$$

By the statement (i) of Theorem $2.3, \mathcal{A}_{J}$ has a nonnegative eigenvalue-eigenvector $\left(l, x_{J}^{*}\right)$ such that

$$
\mathcal{A}_{J}\left(\boldsymbol{x}_{J}^{*}\right)^{m-1}=l\left(\boldsymbol{x}_{J}^{*}\right)^{[m-1]}, \sum_{i \in J}\left(\boldsymbol{x}_{i}^{*}\right)^{m}=1, l \geqslant 0, \boldsymbol{x}_{J}^{*} \geqslant 0 .
$$

Let $x^{*}=\left(0_{I}, x_{J}^{*}\right)$. Then, clearly, $\left(l, x^{*}\right)$ is a nonnegative eigenvalue-eigenvector of $\mathcal{A}$, which contradicts with (b). This completes the proof.

\section{Remark 4}

The statement (b) of Theorem 3.5 does not hold for weakly irreducible tensors. Consider a threeorder two-dimensional tensor $\mathcal{Q}$ given by $q_{111}=1, q_{222}=2, q_{112}=q_{121}=q_{211}=1$ and 
zero elsewhere. By simple computation, we obtain $\mathcal{Q}$ is weakly irreducible and it has a positive eigenvector $\boldsymbol{x}=(1,1)^{T}$ with associated eigenvalue 3 . However, $\mathcal{Q}$ also has an eigenvalue 2 with corresponding eigenvector $\boldsymbol{y}=(0,1)^{T}$. This also means that Problem (3.10) may have more than one KKT pairs for weakly irreducible tensors. In addition, in a similar argument as in Theorem 3.5, we can prove that a nonnegative tensor (not necessarily symmetric) $\mathcal{A}$ is irreducible if and only if the following system has a unique solution $(\lambda, \boldsymbol{x})$ :

$$
\mathcal{A} \boldsymbol{x}^{m-1}=\lambda \boldsymbol{x}^{[m-1]}, \sum_{i=1}^{n} \boldsymbol{x}_{i}^{m}=1, \lambda \geqslant 0, \boldsymbol{x} \geqslant 0,
$$

and $\lambda>0, x>0$.

\subsection{Convex optimization reformulation}

In this subsection, we show that the largest eigenvalue of a symmetric nonnegative tensor is linked to a convex polynomial optimization problem.

\section{Theorem 3.6}

Suppose nonnegative tensor $\mathcal{A}$ is symmetric. If $\boldsymbol{x}^{*}$ is a global solution of Problem (3.10), then $f\left(\boldsymbol{x}^{*}\right)$ is the largest eigenvalue of $\mathcal{A}$ with corresponding eigenvector $\boldsymbol{x}^{*}$. In addition, if $\lambda$ is the largest eigenvalue of $\mathcal{A}$ associated with the eigenvector $\boldsymbol{x}^{*}$ satisfying $\sum_{i=1}^{n}\left(x_{i}^{*}\right)^{m}=1$, then $\boldsymbol{x}^{*}$ is a global solution of Problem (3.10).

\section{Proof}

It follows from Lemma 3.1 that if $\boldsymbol{x}^{*}$ is a global solution of Problem (3.10), then $f\left(\boldsymbol{x}^{*}\right)$ is an eigenvalue of $\mathcal{A}$ with corresponding eigenvector $\boldsymbol{x}^{*}$. Assume to the contrary that $f\left(\boldsymbol{x}^{*}\right)$ is not the largest eigenvalue of $\mathcal{A}$ and $\lambda\left(>f\left(\boldsymbol{x}^{*}\right)\right)$ is the largest eigenvalue of $\mathcal{A}$ associated with the nonnegative eigenvector $\boldsymbol{y}$ satisfying $\sum_{i=1}^{n} y_{i}^{m}=1$. Then, we obtain

$$
\begin{aligned}
\mathcal{A} \boldsymbol{y}^{m-1} & =\lambda \boldsymbol{y}^{[m-1]}, \\
\sum_{i=1}^{n} \boldsymbol{y}_{i}^{m} & =1
\end{aligned}
$$

From (3.11) and (3.13), we have

$$
f(\boldsymbol{y})=\mathcal{A} \boldsymbol{y}^{m}=\boldsymbol{y}^{T}\left(\mathcal{A} \boldsymbol{y}^{m-1}\right)=\lambda \boldsymbol{y}^{T}\left(\boldsymbol{y}^{[m-1]}\right)=\lambda \sum_{i=1}^{n} \boldsymbol{y}_{i}^{m}=\lambda>f\left(\boldsymbol{x}^{*}\right) .
$$

This is a contradiction because $\boldsymbol{x}^{*}$ is a global solution of Problem (3.10). In a similar argument, we can prove that if $\lambda$ is the largest eigenvalue of $\mathcal{A}$ associated with the eigenvector $\boldsymbol{x}^{*}$ satisfying $\sum_{i=1}^{n}\left(\boldsymbol{x}_{i}^{*}\right)^{m}=1$, then $\boldsymbol{x}^{*}$ is a global solution of Problem (3.10).

In the following, we will show that Problem (3.10) can be reformulated as a convex optimization problem and moreover, Problem (3.10) has a unique positive solution if $\mathcal{A}$ is weakly irreducible.

\section{Lemma 3.4}

Suppose the nonnegative tensor $\mathcal{A}$ is symmetric and weakly irreducible. Then, Problem (3.10) has a unique solution $\boldsymbol{x}^{*}$ and $\boldsymbol{x}^{*} \in \operatorname{int}\left(\boldsymbol{P}_{n}\right)$.

Proof

Because $\mathcal{A}$ is weakly irreducible, $f(\boldsymbol{x})$ is an irreducible polynomial function. By Theorem 5.4 [32], this theorem holds. 
Lemma 3.5

Suppose the nonnegative tensor $\mathcal{A}$ is symmetric and weakly irreducible. Then, $\lambda$ is the largest eigenvalue of $\mathcal{A}$ associated with the eigenvector $\boldsymbol{x}^{*}$ satisfying $\sum_{i=1}^{n}\left(\boldsymbol{x}_{i}^{*}\right)^{m}=1$ if and only if $\boldsymbol{x}^{*}$ is the unique solution of Problem (3.10) and $\lambda=f\left(x^{*}\right)$.

Proof

By Lemma 3.4 and Theorem 3.6, this lemma holds.

Let $\boldsymbol{y}=\left(x_{1}^{m}, x_{2}^{m}, \ldots, x_{n}^{m}\right)^{T}$. Then, $f(x)$ can be converted into a homogeneous polynomial form of $\boldsymbol{y}$, and we denote it by $g(\boldsymbol{y})$. Hence, Problem (3.10) can be formulated as the following optimization problem:

$$
\begin{aligned}
\max & g(\boldsymbol{y}) \\
\text { s.t } & \sum_{i=1}^{n} \boldsymbol{y}_{i}=1 \\
& \boldsymbol{y} \geqslant 0 .
\end{aligned}
$$

Theorem 3.7

Suppose the nonnegative tensor $\mathcal{A}$ is symmetric. Then, $g(\boldsymbol{y})$ in Problem (3.28) is a concave function on $\operatorname{int}\left(\boldsymbol{P}_{n}\right)$.

Proof

By Theorem 5.2 [32], this theorem holds.

By Theorem 3.7, Problem (3.28) can be converted into the following convex optimization problem:

$$
\begin{aligned}
\min & -g(\boldsymbol{y}) \\
\text { s.t } & \sum_{i=1}^{n} \boldsymbol{y}_{i}=1 \\
& \boldsymbol{y} \geqslant 0 .
\end{aligned}
$$

\section{Theorem 3.8}

Suppose the nonnegative tensor $\mathcal{A}$ is symmetric. Then, Problem (3.29) is a convex optimization problem. Furthermore, if $\mathcal{A}$ is weakly irreducible, then Problem (3.29) has a unique solution $\boldsymbol{y}^{*}$ and $\boldsymbol{y}^{*} \in \operatorname{int}\left(\boldsymbol{P}_{n}\right)$.

Proof

By Lemma 3.5 and Theorem 3.7, this theorem holds.

\section{ALGORITHMS FOR THE SPECTRAL RADIUS FOR SYMMETRIC NONNEGATIVE TENSORS}

In this section, we discuss two algorithms for computing the spectral radius for symmetric nonnegative tensors. One is a power type algorithm based on Theorem 3.2, and the other is a geometric programming (GP) method due to Theorem 3.3.

\subsection{Power type algorithm}

Given a symmetric nonnegative tensor $\mathcal{A}$ of order $m$ and dimension $n$, we first propose a procedure to compute a partition $\left\{I_{1}, I_{2}, \ldots, I_{k}\right\}$ of $\{1,2, \ldots, n\}$ such that each induced tensor $\mathcal{A}_{I_{i}}, i=$ $1,2, \ldots, k$ is either weakly irreducible or a zero tensor. This procedure is based on Definition 2.3 that the graph of a weakly irreducible tensor is strongly connected.

\section{Procedure 1}

1. Let $J_{1}=\{1,2, \ldots, n\}$ and set $j=1$.

2. If $J_{j}=\emptyset$, then stop. Otherwise, choose an element $i_{1}$ from $J_{j}$, and let $I_{j}:=\left\{i_{1}\right\}$. 
3. Check all elements $a_{i_{1} i_{2} \ldots i_{m}}$ such that $i_{d} \in J_{j}, d=2,3, \ldots, m$. If all these elements are zero, then let $J_{j+1}:=J_{j} \backslash I_{j}$, set $j:=j+1$, and go to Step 2. Otherwise, for all nonzero elements $a_{i_{1} i_{2} \ldots i_{m}}$ and all $d=2,3, \ldots, m, I_{j}:=I_{j} \cup\left\{i_{d}\right\}$ if $i_{d} \notin I_{j}$. Let $K:=\left\{i_{1}\right\}$.

4. If $I_{j} \backslash K=\emptyset$, then let $J_{j+1}:=J_{j} \backslash I_{j}$, set $j:=j+1$, and go to Step 2. Otherwise, go to Step 5.

5. Choose an $i_{k} \in I_{j} \backslash K$, and check all elements $a_{i_{k} i_{2} \ldots i_{m}}$ such that $i_{d} \in J_{j}, d=2,3, \ldots, m$. For all nonzero elements $a_{i_{k} i_{2} \ldots i_{m}}$ and all $d=2,3, \ldots, m, I_{j}:=I_{j} \cup\left\{i_{d}\right\}$ if $i_{d} \notin I_{j}$. Let $K:=K \cup\left\{i_{k}\right\}$, and go to Step 4 .

Procedure 1 can be implemented easily in a computer programming such as MATLAB. To show how Procedure 1 works, we consider the three-order four-dimensional tensor $\mathcal{M}$ given by $m_{222}=2, m_{333}=m_{444}=1, m_{443}=m_{434}=m_{344}=1$, and zero elsewhere. By Procedure 1, a partition of $\{1,2,3,4\}$, can be obtained by the following steps:

Step 1: Let $J_{1}:=\{1,2,3,4\}$ and $j:=1$.

Step 2: Choose 1 from $J_{1}$, and let $I_{1}:=\{1\}$.

Step 3: Check all elements $m_{1 i_{2} i_{3}}, i_{2} \in J_{1}, j_{3} \in J_{1}$. Because they are all zero, let $J_{2}:=J_{1} \backslash I_{1}=$ $\{2,3,4\}$ and $j:=2$.

Step 4: Choose 2 from $J_{2}$, and let $I_{2}:=\{2\}$.

Step 5: Check all elements $m_{2 i_{2} i_{3}}, i_{2} \in J_{2}, j_{3} \in J_{2}$, and we have $m_{222} \neq 0$. So let $I_{2}:=I_{2}$ and $K:=\{2\}$.

Step 6: Because $I_{2} \backslash K=\emptyset$, let $J_{3}:=J_{2} \backslash I_{2}=\{3,4\}$ and $j:=3$.

Step 7: Choose 3 from $J_{3}$, and let $I_{3}:=\{3\}$.

Step 8: Check all elements $m_{3 i_{2} i_{3}}, i_{2} \in J_{3}, j_{3} \in J_{3}$, and we have $m_{344} \neq 0$. So let $I_{3}:=I_{3} \cup\{4\}=$ $\{3,4\}$ and $K:=\{3\}$.

Step 9: Because $I_{3} \backslash K=\{4\}$, we choose 4 from $I_{3} \backslash K$ and check all elements $m_{4 i_{2} i_{3}}, i_{2} \in J_{3}, j_{3} \in$ $J_{3}$. We have $m_{443} \neq 0$ and $m_{434} \neq 0$. Let $I_{3}:=I_{3}$ and $K:=K \cup\{4\}=\{3,4\}$.

Step 10: Because $I_{3} \backslash K=\emptyset$, let $J_{4}:=J_{3} \backslash I_{3}$. Because $J_{4}=\emptyset$, we stop the procedure and obtain $I_{1}=\{1\}, I_{2}=\{2\}$ and $I_{3}=\{3,4\}$, which is a partition of $\{1,2,3,4\}$.

We now state a power-type algorithm for the largest eigenvalue of $\mathcal{A}$ as follows.

\section{Algorithm 4.1}

Step 1. By using Procedure 1, compute a partition $\left\{I_{1}, I_{2}, \ldots, I_{k}\right\}$ of $\{1,2, \ldots, n\}$ such that each induced tensor $\mathcal{A}_{I_{i}}, i=1,2, \ldots, k$ is either weakly irreducible or a zero tensor.

Step 2. For $i=1,2, \cdots, k$, do

If $\mathcal{A}_{I_{i}}$ is a zero tensor, then let $\lambda^{(i)}=0$. Otherwise, compute the largest eigenvalue $\lambda^{(i)}$ of $\mathcal{A}_{I_{i}}$ and a corresponding eigenvector $\boldsymbol{u}^{(i)}$ by using Algorithm 2.1

End

Step 3. Output $\rho(\mathcal{A})=\max _{1 \leqslant i \leqslant k} \lambda^{(i)}$. Suppose $\rho(\mathcal{A})=\lambda^{(i)}$, and let $\boldsymbol{u}$ be defined by

$$
\boldsymbol{u}_{j}=\left(\boldsymbol{u}^{(i)}\right)_{j} \text { if } j \in I_{i} \text {. Otherwise, } \boldsymbol{u}_{j}=0 .
$$

Then, $\boldsymbol{u}$ is an eigenvector associated with the largest eigenvalue $\rho(\mathcal{A})$.

By Theorems 2.4 and 2.5, Algorithm 2.1 can produce the largest eigenvalue and its associated eigenvector for weakly irreducible nonnegative tensors. Therefore, in Step 2 of Algorithm 4.1, the largest eigenvalue $\lambda^{(i)}$ of $\mathcal{A}_{I_{i}}$ and a corresponding eigenvector $\boldsymbol{u}^{(i)}$ can be obtained by using Algorithm 2.1 because $\mathcal{A}_{I_{i}}$ is weakly irreducible. By Theorem 3.2, any weakly reducible symmetric nonnegative tensor can be decomposed into some weakly irreducible tensors. Hence, Algorithm 4.1 can produce the largest eigenvalue and its associated eigenvector for any symmetric nonnegative tensor. To show this, we report our numerical results as follows.

Algorithms 2.1 and 4.1 are implemented in MATLAB (R2008b), and all the numerical computations are conducted using an Intel 3.20-GHz computer with 2 GB of RAM. All test tensors are 
Table I. Numerical results of Algorithms 4.1 and 2.1.

\begin{tabular}{|c|c|c|c|c|c|c|c|c|c|}
\hline \multicolumn{2}{|c|}{ Problem } & \multicolumn{4}{|c|}{ Algorithm 4.1} & \multicolumn{4}{|c|}{ Algorithm 2.1} \\
\hline$n$ & $n p$ & $k$ & err & $\rho$ & сри $(\mathrm{s})$ & $k$ & err & $\rho$ & cpu(s) \\
\hline 30 & 2 & 9 & $1.57 \mathrm{e}-006$ & 51.39 & 0.11 & 100 & $2.24 \mathrm{e}-001$ & 51.39 & 0.41 \\
\hline 60 & 2 & 8 & $1.11 \mathrm{e}-007$ & 200.12 & 0.38 & 100 & $9.47 \mathrm{e}-002$ & 200.07 & 2.17 \\
\hline 90 & 2 & 6 & $9.84 \mathrm{e}-007$ & 451.40 & 0.92 & 100 & $1.43 \mathrm{e}-001$ & 450.67 & 4.77 \\
\hline 120 & 2 & 6 & $7.11 \mathrm{e}-007$ & 802.00 & 2.13 & 100 & $1.69 \mathrm{e}-001$ & 800.57 & 9.30 \\
\hline 150 & 2 & 6 & $3.14 \mathrm{e}-007$ & 1251.52 & 4.08 & 100 & $1.17 \mathrm{e}-001$ & 1250.29 & 18.73 \\
\hline 180 & 2 & 6 & $1.64 \mathrm{e}-007$ & 1799.09 & 7.25 & 100 & $1.33 \mathrm{e}-001$ & 1797.24 & 59.20 \\
\hline 210 & 2 & 6 & $1.36 \mathrm{e}-007$ & 2448.70 & 11.72 & 100 & $6.88 \mathrm{e}-002$ & 2447.93 & 114.67 \\
\hline 240 & 2 & 6 & $9.42 \mathrm{e}-008$ & 3198.34 & 17.50 & 100 & $4.11 \mathrm{e}-002$ & 3198.15 & 171.98 \\
\hline 270 & 2 & 6 & $6.49 \mathrm{e}-008$ & 4050.56 & 25.08 & 100 & $5.77 \mathrm{e}-002$ & 4049.77 & 248.80 \\
\hline 300 & 2 & 6 & $7.51 \mathrm{e}-008$ & 5001.54 & 34.33 & 100 & $8.02 \mathrm{e}-002$ & 4999.91 & 341.02 \\
\hline 30 & 3 & 12 & $2.53 \mathrm{e}-006$ & 50.75 & 0.09 & 100 & $2.34 \mathrm{e}-001$ & 50.62 & 0.42 \\
\hline 60 & 3 & 12 & $1.53 \mathrm{e}-007$ & 201.60 & 0.42 & 100 & $9.90 \mathrm{e}-002$ & 201.35 & 2.17 \\
\hline 90 & 3 & 9 & $1.80 \mathrm{e}-006$ & 450.26 & 1.05 & 100 & $1.17 \mathrm{e}-001$ & 449.42 & 4.75 \\
\hline 120 & 3 & 9 & $1.13 \mathrm{e}-006$ & 803.34 & 2.31 & 100 & $2.04 \mathrm{e}-001$ & 801.02 & 9.38 \\
\hline 150 & 3 & 9 & $3.32 \mathrm{e}-007$ & 1253.10 & 4.52 & 100 & $1.70 \mathrm{e}-001$ & 1250.41 & 18.36 \\
\hline 180 & 3 & 9 & $1.97 \mathrm{e}-007$ & 1797.85 & 7.95 & 100 & $4.30 \mathrm{e}-002$ & 1797.52 & 63.23 \\
\hline 210 & 3 & 9 & $1.42 \mathrm{e}-007$ & 2449.32 & 12.72 & 100 & $6.34 \mathrm{e}-002$ & 2448.21 & 114.52 \\
\hline 240 & 3 & 9 & $1.26 \mathrm{e}-007$ & 3205.06 & 19.06 & 100 & $1.72 \mathrm{e}-001$ & 3200.43 & 171.83 \\
\hline 270 & 3 & 9 & $7.07 \mathrm{e}-008$ & 4057.06 & 27.23 & 100 & $1.15 \mathrm{e}-001$ & 4053.68 & 250.78 \\
\hline 300 & 3 & 9 & $4.66 \mathrm{e}-008$ & 5005.00 & 37.36 & 100 & $7.68 \mathrm{e}-002$ & 5002.75 & 341.73 \\
\hline
\end{tabular}

randomly generated with order $d=3$. Throughout the computational experiments, we use the vector of ones as a starting point for Algorithm 2.1, and we terminate it when one of the following conditions is satisfied:

$$
\text { (1) } k \geqslant 100 ; \text { (2) } \bar{\lambda}_{k}-\underline{\lambda}_{k} \leqslant 10^{-6} \text {; or (3) }\left\|x^{(k)}-H\left(x^{(k)}\right)\right\|_{\infty} \leqslant 10^{-6} \text {. }
$$

Our numerical results are summarized in Table I. In this table, $n$ is the dimension of the randomly generated tensor, and $n p$ denotes the number of the partition of $\{1,2, \ldots, n\} . k$ denotes the number of total iterations needed for Algorithm 2.1, and $c p u$ (s) denotes the total computer time in seconds used to solve the problem. $\rho$ is the largest eigenvalue obtained by these algorithms with a corresponding eigenvector $\boldsymbol{x}_{f}$, and $e r r=\left\|A \boldsymbol{x}_{f}{ }^{m-1}-\rho \boldsymbol{x}_{f}^{[m-1]}\right\|_{\infty}$. The results reported in Table I clearly show that the performance of Algorithm 4.1 is better than Algorithm 2.1 for weakly reducible tensors. Algorithm 4.1 is able to produce the largest eigenvalue for all these randomly generated reducible tensors within 12 iterations. However, Algorithm 2.1 fails to give the largest eigenvalue within 100 iterations for most of test problems.

\subsection{Geometric programming method}

Let $\mathcal{A}$ be a symmetric nonnegative tensor of order $m$ and dimension $n$. By Theorem 3.3, clearly, $\rho(\mathcal{A})$ can be obtained by solving the following optimization problem:

$$
\operatorname{Min}_{\boldsymbol{x} \in \operatorname{int}(\boldsymbol{P})} \operatorname{Max}_{1 \leqslant i \leqslant n} \frac{\left(\mathcal{A} \boldsymbol{x}^{m-1}\right)_{i}}{\boldsymbol{x}_{i}^{m-1}} .
$$

Let

$$
\lambda=\operatorname{Max}_{1 \leqslant i \leqslant n} \frac{\left(\mathcal{A} \boldsymbol{x}^{m-1}\right)_{i}}{\boldsymbol{x}_{i}^{m-1}}
$$

Then, we have

$$
\left(\mathcal{A} \boldsymbol{x}^{m-1}\right)_{i} \leqslant \lambda \boldsymbol{x}_{i}^{m-1}, i=1,2, \ldots, n,
$$


which can also be written as

$$
\sum_{i_{2}, \ldots, i_{m}=1}^{n} a_{i i_{2} \ldots i_{m}} \boldsymbol{x}_{i_{2}} \cdots \boldsymbol{x}_{i_{m}} \lambda^{-1} \boldsymbol{x}_{i}^{1-m} \leqslant 1, i=1,2, \ldots, n .
$$

Hence, (4.30) can be reformulated into the following problem:

$$
\begin{array}{lll}
(G P) \quad \text { Min } & \lambda \\
& \text { s.t. } & \sum_{\substack{i_{2}, \ldots, i_{m}=1 \\
\boldsymbol{x} \in \operatorname{int}(\boldsymbol{P}) .}}^{n} a_{i i_{2} \ldots i_{m}} \boldsymbol{x}_{i_{2}} \cdots \boldsymbol{x}_{i_{m}} \lambda^{-1} \boldsymbol{x}_{i}^{1-m} \leqslant 1, i=1,2, \ldots, n
\end{array}
$$

Problem (GP) is a GP, which is extensively studied in [34,35]. The standard barrier-based interiorpoint method for convex optimization can be applied to (GP) in a straightforward way with a polynomial-time complexity; see [35].

We conclude this paper with a remark on these two algorithms. Recently, GP method has been discussed in [31] for computing the largest eigenvalue for nonnegative tensors, and it is shown in $[31,36]$ that the performance of power type method is better than that of the GP method. The advantage of the GP method is that it has a polynomial-time complexity.

\section{ACKNOWLEDGEMENTS}

The authors are grateful to the anonymous referees for their valuable comments, which led to several improvements of the paper. The work of the first author was supported by the Australian Research Council and the National Natural Science Foundation of China (Grant No. 11171180). The second author's work was supported by the Hong Kong Research Grant Council (Grant Nos PolyU 501808, 501909, 502510, and 502111).

\section{REFERENCES}

1. Kolda TG, Bader BW. Tensor decompositions and applications. SIAM Review 2009; 51:455-500.

2. Kolda TG, Mayo JR. Shifted power method for computing tensor eigenpairs. SIAM Journal on Matrix Analysis and Applications 2011; 32:1095-1124.

3. Van Loan C. Future directions in tensor-based computation and modeling, Workshop Report in Arlington, Virginia at National Science Foundation, February 20-21 2009. http://www.cs.cornell.edu/cv/TenWork/FinalReport.pdf.

4. Chang KC, Pearson K, Zhang T. Perron-Frobenius theorem for nonnegative tensors. Communications in Mathematical Sciences 2008; 6:507-520.

5. Lim L-H. Singular values and eigenvalues of tensors: a variational approach. Proceedings of the IEEE International Workshop on Computational Advances in Multi-Sensor Adaptive Processing (CAMSAP '05, Puerto Vallarta, Mexico), Vol. 1, 2005; 129-132.

6. Qi L. Eigenvalues of a real supersymmetric tensor. Journal of Symbolic Computation 2005; 40:1302-1324.

7. Bloy L, Verma R. On Computing the Underlying Fiber Directions from the Diffusion Orientation Distribution Function. In Medical Image Computing and Computer-Assisted Intervention MICCAI, Vol. 2008. Springer: Berlin / Heidelberg, 2008; 1-8.

8. Qi L, Yu G, Wu EX. Higher order positive semi-definite diffusion tensor imaging. SIAM Journal on Imaging Sciences 2010; 3:416-433.

9. Ng M, Qi L, Zhou G. Finding the largest eigenvalue of a non-negative tensor. SIAM Journal on Matrix Analysis and Applications 2009; 31:1090-1099.

10. Ni Q, Qi L, Wang F. An eigenvalue method for testing the positive definiteness of a multivariate form. IEEE Transactions on Automatic Control 2008; 53:1096-1107.

11. De Lathauwer L, De Moor B, Vandewalle J. On the best rank-1 and rank- $\left(R_{1}, \ldots, R_{N}\right)$ approximation of higher-order tensors. SIAM Journal on Matrix Analysis and Applications 2000; 21:1324-1342.

12. Qi L, Wang F, Wang Y. Z-eigenvalue methods for a global polynomial optimization problem. Mathematical Programming 2009; 118:301-316.

13. Chang KC, Pearson K, Zhang T. On eigenvalue problems of real symmetric tensors. Journal of Mathematical Analysis and Applications 2009; 350:416-422.

14. Chang KC, Qi L, Zhou G. Singular values of a real rectangular tensor. Journal of Mathematical Analysis and Applications 2010; 370:284-294.

15. Friedland S, Gaubert S, Han L. Perron-Frobenius theorem for nonnegative multilinear forms and extensions. Linear Algebra and its Applications 2013; 438:738-749. 
16. Lim L-H. Multilinear pagerank: measuring higher order connectivity in linked objects. The Internet Today and Tomorrow, July, 2005.

17. Bulò SR, Pelillo M. New bounds on the clique number of graphs based on spectral hypergraph theory. In Learning and Intelligent Optimization, Stützle T (ed.). Springer Verlag: Berlin, 2009; 45-48.

18. Cooper J, Dutle A. Spectra of Hypergraphs, 2011. Arxiv preprint arXiv: 1106.4856.

19. Hu S, Qi L. Algebraic connectivity of an even uniform hypergraph. Journal of Combinatorial Optimization 2012; 24:564-579.

20. Collatz L. Einschliessungssatz für die charakteristischen Zahlen von Matrizen (German). Mathematische Zeitschrift 1942; 48:221-226.

21. Varga R. Matrix Iterative Analysis. Prentice-Hall, Inc.: Englewood Cliffs, New Jersey, 1962.

22. Chang KC, Pearson K, Zhang T. Primitivity, the convergence of the NQZ method, and the largest eigenvalue for nonnegative tensors. SIAM Journal on Matrix Analysis and Applications 2011; 32:806-819.

23. Liu Y, Zhou G, Ibrahim NF. An always convergent algorithm for the largest eigenvalue of an irreducible nonnegative tensor. Journal of Computational and Applied Mathematics 2010; 235:286-292.

24. Zhang L, Qi L, Xu Y. Finding the largest eigenvalue of an irreducible nonnegative tensor and linear convergence for weakly positive tensors. Journal of Computational Mathematics 2012; 30:24-33.

25. Zhou G, Caccetta L, Qi L. Convergence of an algorithm for the largest singular value of a nonnegative rectangular tensor. Linear Algebra and its Applications 2013; 438:959-968.

26. Hu S, Huang Z, Qi L. Finding the spectral redius of a nonnegative tensor, 9 November 2011. arXiv: 1111.2138v1 [math.NA].

27. Zhang L, Qi L. Linear convergence of an algorithm for computing the largest eigenvalue of a nonnegative tensor. Numerical Linear Algebra with Applications 2012; 19:830-841.

28. Minc H. Nonnegative Matrices. John Wiley and Sons Inc.: New York, 1988.

29. Pearson KJ. Essentially positive tensors. International Journal of Algebra 2010; 4:421-427.

30. Yang Y, Yang Q. Further results for Perron-Frobenius theorem for nonnegative tensors. SIAM Journal on Matrix Analysis and Applications 2010; 31:2517-2530.

31. Yang Q, Yang Y. Further results for Perron-Frobenius theorem for nonnegative Tensors II. SIAM Journal on Matrix Analysis and Applications 2012; 32:1236-1250.

32. Baratchart L, Berthod M, Pottier L. Optimization of positive generalized polynomials under $l_{p}$ constraints. Journal of Convex Analysis 1998; 5:353-379.

33. Nocedal J, Wright S. Numerical Optimization. Springer-Verlag New York, Inc: New York, 1999.

34. Boyd S, Kim S-J, Vandenberghe L, Hassibi A. A tutorial on geometric programming. Optimization and Engineering 2007; 8:67-127.

35. Boyd S, Vandenberghe L. Convex Optimization. Cambridge University Press: Cambridge, UK, 2004.

36. Zhou G, Caccetta L, Teo KL, Wu SY. Nonnegative polynomial optimization over unit spheres and convex programming relaxations. SIAM Journal on Optimization 2012; 22:987-1008. 